Altai State University

Rcta 3iwlogica Sibirica

Journal of Biology

Founded in 2015
WWW.asu.ru

ISSN 2412-1908

Acta Biologica Sibirica, 2017, 3(4), 6-19

UDC 595.772

\title{
An annotated checklist of Dolichopodidae (Diptera) of Israel and adjacent territories
}

\author{
I.Ya. Grichanov \\ All-Russian Institute of Plant Protection, Podbelskogo 3, St.Petersburg, Pushkin, 196608 Russia \\ E-mail: grichanov@mail.ru
}

\begin{abstract}
The first checklist of the Dolichopodidae of Israel and adjacent territories (Golan Heights and West Bank) is presented. A total of 108 species belonging to 34 genera are listed alphabetically. The references, type locality and general distribution are provided for each species.
\end{abstract}

Key words: Diptera; Dolichopodidae; Palearctic Region; Israel; checklist; fauna

\section{Introduction}

The dolichopodid fauna of the East-Mediterranean countries was until recently poorly studied (Negrobov, 1991). A checklist of long-legged flies of the Caucasus and East Mediterranean Basin as a whole included more than 500 species (Grichanov, 2007a), of which 263 species were known from Romania (Pârvu, 2002). The rapid progress has been made during the last decade in studying fauna of the Russian Caucasus, Bulgaria, Iran, Israel and Turkey. The number of known species has reached recently to more than 220 species for the Russian North-Western Caucasus (Grichanov, 2012), about 190 species for Turkey (Tonguç et al., 2016), about 170 species for Bulgaria (Kechev, Ivanova, 2015) and about 150 species for Iran (Grichanov et al., 2017).

The Israeli fauna of Dolichopodidae has never been generalised in one list. Three species have been described from this country before my works, i.e. Dolichopus syriacus Becker, 1917, Sciapus judaeus Parent, 1932, and Aphrosylus schumanni Negrobov, 1979. Three short lists of the collected material have been also published (Bodenheimer, 1937, and Pârvu, 1996, 1997). F.S. Bodenheimer listed 12 species of Dolichopodidae for "Palestine", giving no information about collectors, collection sites and depository. I consider this paper as dealing with fauna of Israel and adjacent territories. Some of these records need confirmation. In all, 27 species of Dolichopodidae have been recorded from Israel before the beginning of my research in 2000. A. Freidberg (1988) suspected that the dolichopodid fauna of Israel might contain about 100 species.

\section{Annotated List of Species}

For each species we provide references, with the names of taxa used by the respective authors if they differ from those used by us; and type locality and general distribution of species (after Grichanov, 2017). Remarks are provided where deemed necessary.

\section{Acropsilus Mik, 1878}

Acropsilus brevitalus (Parent, 1937)

References: Grichanov, 2007b: 142.

Type locality: Congo-Kinshasa, Eala.

Distribution: Israel; Afrotropical: DR Congo, Tanzania. 


\section{Aphrosylus Haliday, 1851}

Aphrosylus parcearmatus Parent, 1925 References: Grichanov, 2007b: 143.

Type locality: Egypt: Sinai, "Abou-Kir". Distribution: Egypt, Israel, Turkey (Antalya).

Aphrosylus schumanni Negrobov, 1979

References: Negrobov, 1979: 473; Grichanov, 2007b: 143.

Type locality: "Haifa, Beirut, Kandia".

Distribution: Israel, Lebanon, ?Crete ("Kandia").

\section{Arabshamshevia Naglis, 2014}

Arabshamshevia negevensis Grichanov, 2016

References: Grichanov, 2016: 94.

Type locality: Israel: En Mor.

Distribution: Israel.

\section{Argyra Macqart, 1834}

Argyra leucocephala (Meigen, 1824)

References: Grichanov, 2007b: 143.

Type locality: not given.

Distribution: Algeria, Andorra, Austria, Azerbaijan, Belarus, Belgium, Bulgaria, Czech, Denmark, Estonia, Finland, France, Germany, Hungary, Iran, Ireland, Israel, Italy, Latvia, Moldova, Netherlands, Norway, Poland, Romania, Russia (Adygea, Chechnya, Crimea, Krasnodar, Leningrad, Moscow, Novosibirsk, Pskov, Ryazan, Voronezh, Yaroslavl, Urals), Slovakia, Sweden, Switzerland, Tunisia, Turkey (Adiyaman, Antalya), UK, Ukraine (Chernovtsy, Kharkiv).

Argyra vestita (Wiedemann,1817)

References: Grichanov, 2007a: 16 (no material provided).

Type locality: Germany: "bei Kiel”.

Distribution: Austria, Belarus, Belgium, Bulgaria, Czech, Denmark, Estonia, Finland, France, Germany, Hungary, Iran, Ireland, Israel, Italy, Macedonia, Netherlands, Norway, Poland, Romania, Russia (Krasnodar, Moscow, Novosibirsk, Ryazan, Vologda), Slovakia, Sweden, Switzerland, Turkey (Kars, Van), UK.

\section{Asyndetus Loew, 1869}

Asyndetus albifacies Parent, 1929

References: Grichanov, 2013b: 29.

Type locality: Mt. Halaib.

Distribution: Israel, Saudi Arabia; Afrotropical: Hala'ib Triangle (Egypt-Sudan border).

Asyndetus chaetifemoratus Parent, 1925

References: Grichanov, 2013b: 43.

Type locality: Egypt: Baharia Oasis.

Distribution: Egypt, Israel.

Asyndetus separatus (Becker, 1902).

References: Grichanov, 2013b: 44.

Type locality: Egypt: Alexandrien, Fayūm.

Distribution: Algeria, Cyprus, Egypt, Greece, Iraq, Israel, Libya, Spain, Tunisia, ?Tajikistan.

Asyndetus transversalis (Becker, 1907)

References: Grichanov, 2007a: 16 (with question mark; no material provided).

Type locality: Egypt: Alexandrien, Fayūm.

Distribution: Algeria, Egypt, Iraq, ?Israel, Malta, Tunisia.

\section{Campsicnemus Haliday, 1851}

Campsicnemus maculatus Becker, 1918

References: Pârvu, 2008: 345.

Type locality: Italy: "Alassio an der italienischen Riviera".

Distribution: Israel, Italy.

Campsicnemus magius (Loew, 1845)

References: Grichanov, 2007b: 143.

Type locality: Italy: Sicily.

Distribution: Algeria, Austria, Azerbaijan, Belgium, Bosnia and Herzegovina, Bulgaria, Czech, France, Germany, Hungary, Iran, Israel, Italy, Netherlands, Spain, Romania, Russia (Astrakhan, Ekaterinburg, Kabardino-Balkaria, Krasnodar, Rostov, Yakutia), Tajikistan, Turkey (Korucuk), Turkmenistan, UK, Ukraine (Odessa), Uzbekistan; Afrotropical: St. Helena (?introduced). 
Campsicnemus picticornis (Zetterstedt, 1843)

References: Pârvu, 1997: 180.

Type locality: Sweden: "Suecia meridionali et media, in Scania ad Raften; Ostrogothia ad Larketorp; Haradshammar; Holmiam".

Distribution: Austria, Belgium, Czech, Denmark, Estonia, Finland, France, Germany, Hungary, Israel, Italy, N Kazakhstan, Kyrgyzstan, Latvia, Netherlands, Poland, Russia (Birobidzhan, Blagoveshchensk, Buryatia, Kaliningrad, Kamchatka, Karelia, Khabarovsk, Krasnoyarsk, Leningrad, Novosibirsk, Sayan Mts., Yakutia, Vladivostok), Slovakia, Sweden, Turkey (Hakkari), UK, Ukraine (Kherson, Odessa), Uzbekistan.

Campsicnemus simplicissimus Strobl, 1906

References: Grichanov, 2007a: 66 (no material provided).

Type locality: Spain: Algeciras.

Distribution: Abkhazia, Bulgaria, France, Greece: North Aegean, Hungary, Italy, Israel, Russia (Karachai-Cherkessia, Krasnodar, Rostov), Spain, Switzerland, Tajikistan, Turkey (Antalya, Beyşehir lake).

Campsicnemus umbripennis Loew, 1856

References: Grichanov, 2007b: 143.

Type locality: Austria.

Distribution: Abkhazia, Afghanistan, Armenia, Austria, Azerbaijan, Belgium, Bulgaria, Czech, France, Georgia, Germany, Greece incl. North Aegean, Hungary, Iran, Iraq, Israel, Italy, Poland, Portugal, Romania, Russia (Adygea, Alania, Kabardino-Balkaria, Karachai-Cherkessia, Krasnodar, Vladivostok), Slovakia, Spain, Switzerland, Tajikistan, Turkey (Erzurum, Hakkari, Isparta, Kars, Muğla), Turkmenistan, UK.

\section{Chrysotimus Loew, 1857}

Chrysotimus flaviventris (von Roser, 1840)

References: Grichanov, 2007b: 143.

Type locality: not given [Wurttemberg, Germany].

Distribution: Belgium, Bulgaria, Czech, Denmark, France, Germany, Hungary, Israel, Luxembourg, Netherlands, Norway, Poland, Romania, ?Russia, Slovakia, Sweden, Switzerland, UK.

\section{Chrysotus Meigen, 1824}

Chrysotus suavis Loew, 1857

References: Bodenheimer, 1937: 187 ("Palestine”); Pârvu, 1996; Grichanov, 2007b: 144.

Type locality: Germany: “Coln”; Austria: “Neusiedler See in Ungarn”.

Distribution: Afghanistan, Algeria, Armenia, Austria, Azerbaijan, Belgium, Bosnia and Herzegovina, Bulgaria, N China, Croatia, Czech, Egypt, Estonia, Finland, France, Georgia, Germany, Greece (North Aegean), Hungary, Iran, Iraq, Israel, Italy, Kyrgyzstan, Latvia, Lithuania, Middle Asia, Mongolia, Morocco, Netherlands, Norway, Poland, Romania, Russia (Adygea, Alania, Altai Rep., Astrakhan, Blagoveshchensk, Buryatia, Irkutsk, Kabardino-Balkaria, Kamchatka, Khabarovsk, Krasnodar, Krasnoyarsk, Kursk, Leningrad, Lipetsk, Magadan, Novosibirsk, Pskov, Rostov, Ryazan, Sakhalin, Tatarstan, Voronezh, Yakutia), Serbia, Slovakia, Spain (Canary Is), Sweden, Switzerland, Turkey (Adiyaman, Antalya, Antakya, Artvin, Gaziantep, Kizildere, Sanliurfa), UK, Ukraine (Cherkasy, Kherson, Odessa).

\section{Diaphorus Meigen, 1824}

Diaphorus gredleri Mik, 1880

References: Grichanov, 2007b: 144.

Type locality: Italy: “Calvarienberge bei Bozen [= Bolzano] in Südtirol”.

Distribution: Austria, France, Israel, Italy, Spain, Switzerland, Turkey (Kars), Tunusia.

Diaphorus hoffmannseggi Meigen, 1830

References: Grichanov, 2007a: 20 (no material provided).

Type locality: not given.

Distribution: Austria, Belgium, Czech, Denmark, Finland, France, Germany, Hungary, Israel, Italy, Latvia, Netherlands, Poland, Romania, Russia (Leningrad, Moscow, North Caucasus, Ural, Voronezh), Slovakia, Sweden, Switzerland, Turkey (Zonguldak), UK.

Diaphorus nigricans Meigen, 1824

References: Grichanov, 2007b: 144.

Type locality: Germany.

Distribution: Abkhazia, Austria, Belarus, Belgium, China (Xinjiang), Czech, Denmark, Estonia, Finland, France, Germany, Greece, Hungary, India (Kashmir), Ireland, Italy, Israel, Netherlands, Norway, Poland, Romania, Russia (Karelia, Krasnodar, Leningrad, Moscow, Murmansk, Yakutia), Spain, Sweden, Switzerland, UK; Afrotropical: DR Congo; Oriental: China (Chongqing, Guizhou, Henan, Sichuan, Yunnan, Zhejiang); Nearctic: USA, Mexico; Neotropical: Argentina, Dominica, Brazil, Mexico.

Diaphorus varifrons Becker, 1918

References: Grichanov, 2007a: 21 (no material provided). 
Type locality: Tunisia, Mehtia (Bel-Mehtia)

Distribution: ?Israel, Turkey (Muğla), Tunisia.

\section{Dolichopus Latreille, 1796}

Dolichopus callosus Becker, 1902

References: Grichanov, 2007b: 144.

Type locality: Egypt: Suez.

Distribution: Israel, Egypt, Kyrgyzstan.

Dolichopus diadema Haliday, 1832

References: Grichanov, 2007b: 144.

Type locality: Ireland: Holywood.

Distribution: Austria, Azerbaijan, Belgium, Bulgaria, China, Denmark, Estonia, Finland, France, Germany, Greece, Ireland, Israel, Italy, Kazakhstan, Kyrgyzstan, Mongolia, Netherlands, Norway, Poland, Portugal, Romania, Russia (Rostov), Spain, Sweden, Turkey (Muğla), UK, Ukraine.

Dolichopus excisus Loew, 1859

References: Grichanov, 2007a: 24 (no material provided), but see Pârvu, 1996.

Type locality: Germany: "in alien Theilen Deutschlands".

Distribution: Abkhazia, Armenia, Austria, Azerbaijan, Belarus, Belgium, Bulgaria, Czech, France, Germany, Hungary, Iran, Israel, Italy, Netherlands, Poland, Romania, Russia (Adygea, Chechnya, Crimea, Kabardino-Balkaria, Krasnodar, Voronezh, “Siberia”), Slovakia, Spain, Tajikistan, Turkey (Antalya, Burdur, Muğla), Turkmenistan.

Dolichopus griseipennis Stannius, 1831

References: Grichanov, 2007b: 144.

Type locality: France: Lyon.

Distribution: Algeria, ?Armenia, Azerbaijan, Austria, Belgium, Bulgaria, Cyprus, Czech, Denmark, Egypt, Estonia, Finland, France, Georgia, Germany, Greece incl. Crete, Hungary, Iran, Ireland, Israel, Italy, N Kazakhstan, Lithuania, Luxembourg, Morocco, Netherlands, Norway, Poland, Romania, Russia (Adygea, Krasnodar, Moscow, "Siberia"), Serbia, Slovakia, Spain incl. Balearic Is., Sweden, Switzerland, Tunisia, Turkey (Sinop), UK, "Middle Asia”.

Dolichopus nitidus Fallén, 1823

References: Bodenheimer, 1937: 187 (“Palestine”).

Type locality: not given.

Distribution: Austria, Belarus, Belgium, Bulgaria, Czech, Denmark, Estonia, Finland, France, Germany, Hungary, Ireland, ?lsrael, Italy, Japan, Kazakhstan, Netherlands, Norway, Poland, Romania, Russia (Altai Rep., Karelia, Khabarovsk, Khanty-Mansi, Krasnodar, Moscow, Novgorod, Ryazan, Tatarstan, Voronezh, Vladivostok), Slovakia, Spain, Sweden, Switzerland, UK, Ukraine (Odessa); Oriental: China (Henan, Shanghai).

Dolichopus nivalis Vaillant, 1973

References: Grichanov, 2007a: 27 (no material provided).

Type locality: France: 1 'Isere, Prarion, Pats Vanoise.

Distribution: ?lsrael; France.

Dolichopus perversus Loew, 1871

=Dolichopus subimmaculatus Kazerani, Pollet, Khaghaninia, 2017.

References: Pârvu, 1996: 280 (as Dolichopus immaculatus, misidentification); Grichanov, 2007b: 144; Kazerani et al., 2017: 117 (as Dolichopus subimmaculatus).

Type locality: Tajikistan: "Zarawschan Thal [=Zeravshan valley], Turkestan".

Distribution: Abkhazia, Armenia, Iran, Israel, Kazakhstan, Kyrgyzstan, Tajikistan, Turkey (Antalya).

Dolichopus sabinus Haliday, 1838

References: Pârvu, 1996: 280; Grichanov, 2007b: 145.

Type locality: Ireland: Killarney, Tarbert.

Distribution: Abkhazia, Austria, Azerbaijan, Belgium, Bulgaria, Czech, Denmark, Estonia, Finland, France, Germany, Greece, Hungary, Ireland, Israel, Italy, Latvia, Netherlands, Norway, Poland, Romania, Russia (Kabardino-Balkaria, Saratov), Slovakia, Spain, Sweden, Turkey (Antalya), UK, Ukraine; Afrotropical: Tanzania.

Dolichopus siculus Loew, 1859

References: Pârvu, 1996: 280; Grichanov, 2007b:145.

Type locality: Italy: Sidy.

Distribution: Bulgaria, France, Iran, Israel, Golan Heights, Italy.

Dolichopus syriacus Becker, 1917

References: Becker, 1917: 159.

Type locality: Israel: “Haifa, Syrien”.

Distribution: Israel. 
References: Grichanov, 2008: 134.

Type locality: France: Bastia, Corsica.

Distribution: France, Israel, Morocco; Afrotropical: St. Helena.

Epithalassius susmani Grichanov, 2008

References: Grichanov, 2008: 134.

Type locality: Israel: Achziv.

Distribution: Israel.

\section{Hercostomus Loew, 1857}

Hercostomus chetifer (Walker, 1849)

References: Grichanov, 2007b: 145.

Type locality: England.

Distribution: Algeria, Austria, Belgium, Bosnia and Herzegovina, Croatia, Czech, Finland, France, Germany, Greece, Hungary, Israel, Italy, Luxembourg, Netherlands, Norway, Poland, Romania, Russia (Adygea, Krasnodar, Moscow), Slovakia, Spain, Sweden, Switzerland, Turkey (Antalya), UK, Ukraine; Nearctic: Canada, USA: Alberta, Ontario, Michigan, Tennessee, Quebec, Pennsylvania, New York, Connecticut, New Jersey, North Carolina; Oriental: India.

Hercostomus convergens (Loew, 1857)

References: Grichanov, 2007b: 145.

Type locality: Italy; Austria.

Distribution: Austria, Azerbaijan, Bulgaria, France, Germany, Hungary, Israel, Italy, Poland, Romania, S Russia (Krasnodar, Voronezh), Spain, Turkey (Kars, Muğla), Ukraine (Odessa).

Hercostomus golanensis Grichanov, 2015

References: Grichanov, 2015: 427.

Type locality: Israel: Golan (Heights), Qatzrin.

Distribution: Israel, Golan Heights.

Hercostomus longiventris (Loew, 1857)

References: Grichanov, 2007a: 34 (no material provided).

Type locality: Austria: Murzzuschlag.

Distribution: Austria, Belgium, Croatia, Czech, France, Georgia, Germany, Greece, Hungary, Iran, Israel, Italy, Morocco, Netherlands, Poland, Romania, Russia (Alania, Kabardino-Balkaria, Krasnodar), Switzerland, Tajikistan, Turkey (Artvin, Muğla).

\section{Hydrophorus Fallén, 1823}

Hydrophorus balticus (Meigen, 1824)

References: Pârvu, 1996: 284; Grichanov, 2007b:145.

Type locality: Germany: Hamburg.

Distribution: Afghanistan, Algeria, Austria, Azerbaijan, Belgium, Bosnia and Herzegovina, Bulgaria, Cyprus, Czech, Denmark, Estonia, Finland, France, Georgia, Germany, Greece, Hungary, Iran, Ireland, Israel, Italy, Mongolia, Morocco, Netherlands, Norway, Poland, Romania, Russia (Adygea, Alania, Baikal, Chechnya, Kabardino-Balkaria, Karachai-Cherkessia, Krasnodar, Leningrad, Moscow, Voronezh, Yakutia), Slovakia, Spain, Sweden, Switzerland, Turkey (Antalya, Aydin, Denizli, Isparta, Kars, Muğla, Rize), UK, Ukraine; Afrotropical: South Africa, St. Helena.

Remark. The species was most probably mentioned by Bodenheimer (1937: 187) for "Palestine" under the name Hydrophorus viridis (Meigen, 1824) (here excluded from the fauna of Israel).

Hydrophorus praecox (Lehmann, 1822)

References: Pârvu, 1996: 284; Pârvu, 1997: 179; Grichanov, 2007b:145.

Type locality: Germany: Hamburg.

Distribution: Abkhazia, Austria, Belgium, Bulgaria, China, Cyprus, Czech, Denmark, Egypt, Estonia, Finland, France, Georgia, Germany, Greece, Hungary, Iraq, Iran, Ireland, Israel, Kazakhstan, Mongolia, Netherlands, Norway, Poland, Portugal (Azores), Romania, Russia (incl. Caucasus: Chechnya, Kabardino-Balkaria, Krasnodar, Stavropol), Slovakia, Spain, Sweden, Switzerland, Turkey (Antalya, Bolu), UK, Ukraine; Oriental: China, India; Australian: Australia, French Polynesia, New Zealand; Afrotropical: Aldabra, Cape Verda Is., Ethiopia, Kenya, Tanzania, Mauritius, Rodriguez, South Africa, Namibia, Angola, Botswana, Gambia, Senegal, South Arabia, Mauritania, St Helena, Nigeria; Neotropical: Chile.

Remark. The species was most probably mentioned by Bodenheimer (1937: 187) for "Palestine" under the name Hydrophorus litoreus Fallén, 1823 (here excluded from the fauna of Israel).

\section{Lamprochromus Mik, 1878}

Lamprochromus kowarzi Negrobov et Tshalaja, 1988

References: Grichanov, 2007a: 67 (as Lamprochromus bifasciatus, misident.); Grichanov, 2007b: 146 (as Lamprochromus speciosus, in part); Grichanov, Ahmadi, 2017: 8.

Type locality: Slovakia: Lozonez. 
Distribution: Azerbaijan, Israel, Russia (Chechnya), Slovakia.

Lamprochromus speciosus (Loew, 1871)

References: Bodenheimer, 1937: 187 (“Palestine”); Grichanov, 2007b: 146; Grichanov, Ahmadi, 2017: 9.

Type locality: Tajikistan: "Sarawschan Thal".

Distribution: Bulgaria, Egypt, France, Greece, Hungary, Iran, Iraq, Israel, Romania, Russia (Krasnodar), Spain (Canary Is.), Uzbekistan, Tajikistan, Turkey (Muğla), Ukraine (Odessa).

\section{Liancalus Loew, 1857}

Liancalus virens (Scopoli, 1763)

References: Grichanov, 2007b: 146.

Type locality: not given ["Carnioliae indigena", Slovenia].

Distribution: Abkhazia, Algeria, Austria, Azerbaijan, Belgium, Bulgaria, Cyprus, Czech, Denmark, Finland, France, Georgia; Germany, Greece incl. Crete, Hungary, Iran, Ireland, Israel, Golan Heights, West Bank, Italy, S Kazakhstan, Kyrgyzstan, Luxembourg, Morocco, Netherlands, Norway, Poland, Portugal incl. Madeira, Romania, Russia (Crimea, Krasnodar, Leningrad, "Siberia”), Slovakia, Slovenia, Spain, Sweden, Switzerland, Tajikistan, Tunisia, Turkey (Hakkari, Muğla), UK.

\section{Medetera Fischer von Waldheim, 1819}

Medetera dendrobaena Kowarz, 1877

References: Bodenheimer, 1937: 187 (“Palestine”).

Type locality: Austria; Germany.

Distribution: Austria, Belgium, Czech, France, Germany, Greece, Hungary, Iraq, Ireland, Israel, Italy, Netherlands, Spain, UK.

Medetera diadema (Linnaeus, 1767)

References: Grichanov, 2007b: 146.

Type locality: Europe.

Distribution: Abkhazia, Algeria, Austria, Belarus (Minsk, Grodno), Belgium, Bulgaria, Croatia, Czech, Denmark, Egypt, Estonia, France, Germany, Greece incl. Crete and North Aegean, Hungary, Iran, Israel, Golan Heights, Italy, N Kazakhstan, "Middle Asia", Latvia, Netherlands, Poland, Romania, Russia (Alania, Adygea, Altai Rep., Chechnya, Crimea, Krasnodar, Kursk, Leningrad, Lipetsk, Moscow, Orenburg, Rostov, Ryazan, Samara, Voronezh), Slovakia, Spain, Sweden, Tunisia, Turkey (Izmir-Yenifoça, "Bujuk-Dere bei Constantinopel"), UK, Ukraine (Kharkiv, Kherson, Odessa, Carpathia); Nearctic: Washington, California, New Hampshire, Massachusetts, Connecticut, New Jersey, Rhode Island; ?Afrotropical: ?Guinea.

Medetera flavipes Meigen, 1824

References: Grichanov, 2007b: 146.

Type locality: not given.

Distribution: Algeria, Azerbaijan, Belgium, Croatia, Czech, Egypt, France, Germany, Greece, Iran, Israel, Golan Heights, Italy, Malta, Morocco, Poland, Portugal, Russia (Crimea, Krasnodar), Serbia, Spain incl. Canary Is., Syria, Turkey (Antalya-Side, Aydin, Izmir, Muğla, Adiyaman), UK, Ukraine.

Medetera media Parent, 1925

References: Grichanov, 2007a: 50 (no material provided).

Type locality: Tunisia.

Distribution: Egypt, ?lsrael, W Kazakhstan, ?Russia, Turkmenistan, Tunisia.

Medetera micacea Loew, 1857

References: Grichanov, 2007b: 147.

Type locality: not given [Europe].

Distribution: Austria, Belgium, Bulgaria, China, Denmark, Estonia, France, Germany, Greece, Hungary, Israel, West Bank, Italy, Kazakhstan, Malta, Mongolia, Netherlands, Norway, Poland, Romania, Russia (Adygea, Crimea, Krasnodar, Leningrad, Omsk, Voronezh, Yakutia), Slovakia, Spain, Sweden, UK, Ukraine (Cherkasy), Uzbekistan.

Medetera muralis Meigen, 1824

References: Grichanov, 2007b: 147.

Type locality: Germany: Hamburg.

Distribution: Abkhazia, Austria, Azerbaijan, Belarus, Belgium, Bosnia and Herzegovina, Czech, Denmark, Estonia, Finland, France, Germany, Hungary, Ireland, Italy, Israel, Netherlands, Norway, Poland, Romania, Russia (Adygea, Alania, Kabardino-Balkaria, Krasnodar, Moscow), Slovakia, Sweden, Turkey (Antalya, Denizli), UK.

Medetera pallipes (Zetterstedt, 1843)

References: Grichanov, 2007b: 147.

Type locality: Scania, "in Ostrog ad Wadstena; Botnia orientali ad Johannis Ro prope Tormea" [Sweden; Denmark].

Distribution: Austria, Belgium, Czech, Denmark, Egypt, Estonia, Finland, France, Georgia, Germany, Greece, Hungary, Iran, Israel, Golan Heights, Morocco, Netherlands, Norway, Poland, Romania, Russia (Crimea, Kabardino-Balkaria, 
Krasnodar, Leningrad, Novgorod, Ryazan, Stavropol, Voronezh), Slovakia, Spain, Switzerland, Sweden, Turkey (Antalya, Hakkari, Kars), UK, Ukraine (Kherson).

Medetera perfida Parent, 1932

References: Grichanov, 2007a: 52 (no material provided).

Type locality: Austria: Sölden, Ötztal.

Distribution: Austria, Belgium, France, Germany, Israel, Russia (Adygea, Chechnya, Crimea), Turkey (Usak), Ukraine (Kherson), "North, Central and South Caucasus".

Medetera petrophila Kowarz, 1877

References: Grichanov, 2007b: 147.

Type locality: Kärnten, Gastein, Ober-Italien [Austria; Italy].

Distribution: Austria, Belgium, Bulgaria, Czech, Denmark, France, Germany, Greece incl. North Aegean, Hungary, Ireland, Israel, Golan Heights, Italy, Morocco, Netherlands, Poland, Russia (Krasnodar, Rostov), Slovakia, Spain, Sweden, Switzerland, UK.

Medetera plumbella Meigen, 1824

References: Grichanov, 2007b: 147.

Type locality: Germany: Berlin.

Distribution: Armenia, Austria, Belgium, China, Czech, Denmark, Estonia, Finland, France, Germany, Hungary, Israel, Italy, Kazakhstan, Netherlands, Norway, Poland, Russia (Crimea, Irkutsk, Ryazan, Tatarstan, Voronezh), Slovakia, Sweden, Turkey (Kars).

Medetera striata Parent, 1927

References: Grichanov, 2007b: 147.

Type locality: France: Bois de Linghem aux environs d'Aire-sur-la Lys.

Distribution: Belgium, Estonia, Finland, France, Israel, Lithuania, Norway, Poland, Russia (Adygea, Krasnodar, Murmansk, Novosibirsk), Sweden, UK.

Medetera truncorum Meigen, 1824

References: Grichanov, 2007b: 147.

Type locality: Germany: Hamburg.

Distribution: Algeria, Austria, Azerbaijan, Belgium, Czech, Croatia, Denmark, Egypt, Estonia, Finland, France, Germany, Greece, Hungary, Iran, Ireland, Israel, Luxembourg, Netherlands, Norway, Poland, Portugal incl. Azores, Russia (Chechnya, Crimea, Krasnodar, Yakutia), Slovakia, Spain, Sweden, Switzerland, Turkey (Adiyaman, Gaziantep, Hatay, Isparta, Mersin, Muğla, Sanliurfa), UK, Ukraine (Kharkiv); Nearctic: British Columbia, Wyoming, Oregon.

\section{Melanostolus Kowarz, 1884}

Melanostolus nigricilius (Loew, 1871)

References: Pârvu, 1997: 180.

Type locality: Tajikistan.

Distribution: Bulgaria, China, France, Germany, Hungary, Israel, Mongolia, Romania, Russia (Yakutia), Tajikistan.

\section{Micromorphus Mik, 1878}

Micromorphus aereus (Vaillant, 1953)

References: Grichanov, 2007b: 148.

Type locality: Algeria: Tassili du n'Ajjer.

Distribution: Algeria, Egypt, Israel.

Micromorphus albipes (Zetterstedt, 1843)

References: Bodenheimer, 1937: 187 (“Palestine”); Grichanov, 2007b: 148.

Type locality: Sweden: Ostrogoths; Larketorp.

Distribution: Algeria, Austria, Belgium, Bulgaria, China, Croatia, Czech, Denmark, Egypt, France, Germany, Greece, Hungary, Ireland, Iraq, Israel, West Bank, Italy, Mongolia, Morocco, Netherlands, Poland, Portugal (Azores), Romania, Russia (Krasnodar, Leningrad), Slovakia, Spain, Sweden, Switzerland, Turkey (Korucuk, Kizildere, Muğla), UK, Ukraine (Kherson, Odessa); Oriental, Australasian, Nearctic, Neotropical Regions.

\section{Microphorella Becker, 1909}

Microphorella ebejeri Gatt, 2012

References: Gatt, 2012: 288.

Type locality: Israel: Bor Mashash.

Distribution: Israel.

\section{Neurigona Rondani, 1856}

Neurigona meironensis Grichanov, 2010

References: Grichanov, 2010: 252.

Type locality: Israel: Mt. Meiron. 
Distribution: Israel.

\section{Ortochile Latreille, 1809}

Ortochile nigrocoerulea Latreille, 1809

References: Pârvu, 1997: 180; Grichanov, 2007b: 148.

Type locality: France: Paris.

Distribution: Algeria, Austria, Bulgaria, Croatia, ?Denmark, France, Greece incl. North Aegean Is., Hungary, Israel, Italy, Morocco, Poland, Spain incl. Balearic Is., Sweden, Syria, Tunisia, Turkey (Aydin, Izmir), UK.

\section{Orthoceratium Schrank, 1803}

Orthoceratium lacustre (Scopoli, 1763

References: Grichanov, 2007a: 44 (no material provided).

Type locality: "Carnioliae indigena” [Slovenia].

Distribution: Algeria, Austria, Azerbaijan, Belgium, Bulgaria, Cyprus, Denmark, Finland, France, Germany, Greece incl. North Aegean, Iran, Ireland, ?Israel; Italy, Netherlands, Portugal incl. Madeira, Russia (Crimea), Slovenia, Spain, Tunisia, Turkey (Afyonkarahisar, Kutahya), UK; Afrotropical: Tanzania.

\section{Poecilobothrus Mik, 1878}

Poecilobothrus basilicus (Loew, 1869)

References: Grichanov, 2007b: 149.

Type locality: Italy: Sicily.

Distribution: Azerbaijan, Iran, Israel, Golan Heights, Italy, Turkey (Adiyaman).

Poecilobothrus principalis (Loew, 1861)

References: Grichanov, 2007a: 38 (no material provided).

Type locality: Poland: Meseritz [=Miedzyrzecz]; Netherlands.

Distribution: Austria, Belgium, Bulgaria, Czech, France, Germany, Greece, Hungary, Israel, Italy, Netherlands, Poland, Romania, Russia (Adygea, Krasnodar), Spain, Turkey (Kutahya), UK, Ukraine (Kharkiv, Kherson, Odessa).

\section{Rhaphium Meigen, 1803}

Rhaphium brevicorne Curtis, 1835

References: Negrobov et al., 2013: 602.

Type locality: England: Isle of Wight.

Distribution: Algeria, Belgium, France incl. Corsica, Germany, Greece incl. Crete and North Aegean, Iran, Iraq, Ireland, Israel, Italy, Lebanon, Netherlands, Russia (Krasnodar), Spain incl. Canary Is., Sweden, Tajikistan, Turkey, UK.

Rhaphium caliginosum Meigen, 1824

References: Grichanov, 2007b: 149.

Type locality: not given.

Distribution: Algeria, Armenia, Austria, Azerbaijan, Bosnia and Herzegovina, Bulgaria, Denmark, Estonia, Finland, France, Germany, Greece, Israel, Golan Heights, Italy, Latvia, Morocco, Netherlands, Norway, Romania, Russia (Adygea, Kabardino-Balkaria, Kaliningrad, Karachai-Cherkessia, Krasnodar, Krasnoyarsk, Kursk, Leningrad, Moscow, Murmansk, Pskov, Rostov, Stavropol, Voronezh), Serbia, Sweden, Switzerland, Syria, Turkey (Burdur, İzmir), UK, Ukraine (Kherson, Odessa).

\section{Sciapus Zeller, 1842}

Sciapus albifrons (Meigen, 1830)

References: Bodenheimer, 1937: 187 ("Palestine").

Type locality: not given.

Distribution: Austria, Belgium, Czech, Estonia, Finland, France, Germany, Hungary, Latvia, Lithuania, Netherlands, Israel, Poland, Romania, Russia (Karachai-Cherkessia, Leningrad, Moscow, Novosibirsk, Pskov, Ryazan, Voronezh), Slovakia, Turkey (Buharkent), Ukraine (Kherson).

Sciapus contristans (Wiedemann, 1817)

References: Grichanov, 2007a: 62 (doubtful record).

Type locality: Holstein.

Distribution: Austria, ?Belarus, Belgium, Bulgaria, Croatia, Czech, Egypt, Estonia, France, Germany, Hungary, ?lsrael, Italy, Netherlands, Poland, Romania, ?Russia, Slovakia, Spain, UK, ?Ukraine.

Sciapus euchromus (Loew, 1857)

References: Grichanov, Negrobov, 2014: 13.

Type locality: Not given.

Distribution: Bulgaria, Hungary, Israel (Golan Heights).

Sciapus flavicinctus (Loew, 1857)

References: Grichanov, Negrobov, 2014: 14 (doubtful record). 
Type locality: Turkey: "bei Constantinopel".

Distribution: Azerbaijan, Bulgaria, Denmark, France, Germany, Greece incl. Crete, Hungary, Iran, ?lsrael, Italy, Romania, S Russia (Krasnodar, North Ossetia), Slovakia, Turkey (Adana, Istanbul).

Sciapus freidbergi Grichanov et Negrobov, 2014

References: Grichanov, Negrobov, 2014: 35.

Type locality: Israel: Maagan Michael.

Distribution: Israel.

Sciapus glaucescens (Loew, 1856)

References: Grichanov, 2007b: 149.

Type locality: Egypt.

Distribution: Abkhazia, Bulgaria, Croatia, Egypt, France, Israel, Italy, Morocco, Portugal (Madeira, Azores), Spain (Canary Is.), Russia (Crimea), Turkey (Mersin).

Sciapus heteropygus Parent, 1926: Enc. ent. (B II) Dipt. 3: 30 [Sciopus]

References: Grichanov, 2007b: 149.

Type locality: France: Ardennes, "Mézières".

Distribution: Czech, Denmark, France, Germany, Greece, Hungary, Israel, Romania, Slovakia, Spain, Switzerland, Turkey (Muğla), UK.

Sciapus judaeus Parent, 1932

References: Grichanov, 2007b: 149.

Type locality: "Palestine: Jerusalem, Scopusberg”.

Distribution: Cyprus, Israel.

Sciapus longitarsis Grichanov et Negrobov, 2014

References: Grichanov et Negrobov, 2014: 31.

Type locality: Israel: Haifa.

Distribution: Israel, Golan Heights, West Bank.

Sciapus maurus Parent, 1930

References: Grichanov, Negrobov, 2014: 21 (doubtful record).

Type locality: Algeria: "Jean Bart, d'Alger".

Distribution: Algeria, ?Bulgaria, ?lsrael, Tunisia, Turkey (Antalya-Side)

Sciapus occidasiaticus Grichanov et Negrobov, 2014

References: Grichanov, Negrobov, 2014: 44.

Type locality: Israel: Ein Hajla.

Distribution: Israel, West Bank.

Sciapus opacus (Loew, 1866)

References: Grichanov, 2007a: 63 (doubtful record).

Type locality: Italy: Sicily.

Distribution: Bulgaria, Greece, ?Israel, Italy, Spain, Tunisia, "Yugoslavia".

Sciapus pallens (Wiedemann, 1830)

References: Bodenheimer, 1937: 187 (“Palestine").

Type locality: USA: New York.

Distribution: Belgium, Bulgaria, Croatia, France, Greece (Crete), Israel, Italy, Netherlands, Portugal (Azores), Spain, Ukraine (Kherson); Nearctic: USA: Michigan, New York, Massachusetts to Maryland, District of Columbia, and North Carolina.

Sciapus vicinus Parent, 1925

References: Bodenheimer, 1937: 187 ("Palestine").

Type locality: Egypt: Rafa, Sinai.

Distribution: Algeria, Egypt, Israel.

\section{Sybistroma Meigen, 1824}

Sybistroma golanica (Grichanov, 2000)

References: Grichanov, 2000: 273.

Type locality: Israel: Tel Dan.

Distribution: Israel, Golan Heights.

Sybistroma impar (Rondani, 1843)

References: Grichanov, 2000: 274.

Type locality: Italy.

Distribution: Bulgaria, Greece, Hungary, Iran, Israel, Italy, Romania, Russia (Chechnya, Krasnodar), Turkey (Adiyaman, Antalya).

Sybistroma israelensis (Grichanov, 2000)

References: Grichanov, 2000: 270.

Type locality: "Israel, W Nemrod". 
Distribution: Israel, Golan Heights.

Sybistroma nodicornis Meigen, 1824

References: Grichanov, 2007a: 39 (no material provided).

Type locality: not given.

Distribution: Austria, Belgium, Bulgaria, Czech, Egypt, France, Germany, Greece, Hungary, Iran, Iraq, ?Israel, Italy, Netherlands, ?S Russia, Romania, Serbia, Slovakia, Switzerland, Turkey (Afyonkarahisar, Uşak).

Sybistroma occidasiatica Grichanov et Kazerani, 2014

References: Grichanov, Kazerani, 2014: 575.

Type locality: Turkey: near Manavgat.

Distribution: Iran, Israel, Golan Heights, Turkey (Antalya).

Sybistroma sinaiensis (Grichanov, 2000)

References: Grichanov, 2000: 272; Pârvu, 2008: 347.

Type locality: Egypt: Sinai, Wadi Hibran.

Distribution: Egypt (Sinai Mts.), Israel.

\section{Sympycnus Loew, 1857}

Sympycnus simplicipes Becker, 1908

References: Pârvu, 1997: 180; Grichanov, 2007b: 150.

Type locality: Spain: Canary Is., Teneriffe.

Distribution: Abkhazia, Austria, Azerbaijan, Czech, Egypt, France, Germany, Greece incl. Crete, Iran, Iraq, Israel, Italy, Japan, N Kazakhstan, Korea, Kyrgyzstan, Russia (Adygea, Krasnodar), Spain incl. Canary Is., Tajikistan, Turkey (Afyonkarahisar, Kütahya, Uşak), Uzbekistan; Afrotropical: DR Congo, Kenya, South Africa; Oriental: China (Taiwan), India (Kashmir, West Bengal).

\section{Syntormon Loew, 1857}

Syntormon denticulatus (Zetterstedt, 1843)

=Syntormon pumilus Parent, 1925 (nec Meigen, 1824; misidentification).

References: Bodenheimer, 1937: 187 (“Palestine”); Pârvu, 1996: 284 (as Syntormon pumilus); Pârvu, 1997: 180 (as Syntormon pumilus); Grichanov, 2007b: 150.

Type locality: Sweden: Scania.

Distribution: Palaearctic: Abkhazia, Afghanistan, Armenia, Azerbaijan, Belorussia, Bosnia and Herzegovina, Bulgaria, Estonia, Finland, France, Germany, Greece, Iran, Israel, Golan Heights, West Bank, Italy, Kyrgyzstan, Norway, Poland, Romania, Russia (Adygea, Alania, Kabardino-Balkaria, Karelia, Leningrad, Moscow, Murmansk, Stavropol), Sweden, Tajikistan, Turkey, UK, Ukraine, "North Africa".

Syntormon macula mediterraneus Grichanov, 2013

References: Grichanov, 2013a: 18.

Type locality: "Israel: Baniass [Panyas]".

Distribution: Greece (Rhodes), Iran, Israel, Golan Heights.

Syntormon miki Strobl, 1899

References: Grichanov, 2013a: 10 (doubtful record).

Type locality: Spain: Algeciras.

Distribution: Bulgaria, Czech, France incl. Corsica, Greece incl. Crete, Ireland, ?Israel, Italy, Morocco, Poland, Portugal, Spain, "Yugoslavia", Sweden, Tunisia, UK.

Syntormon pallipes (Fabricius, 1794)

References: Pârvu, 1996: 284; Grichanov, 2001: 186, 2007b: 150.

Type locality: Germany.

Distribution: Abkhazia, Afghanistan, Algeria, Armenia, Austria, Azerbaijan, Belgium, Bosnia and Herzegovina, Bulgaria, China, Croatia, Czech, Denmark, Egypt, Estonia, Finland, France, Georgia, Germany, Greece incl. Crete, Hungary, Iceland, Iran, Iraq, Ireland, Israel, Italy, Jordan, Kyrgyzstan, Latvia, Morocco, Netherlands, Norway, Poland, Portugal incl. Madeira, Azores, Romania, Russia (Adygea, Alania, Chechnya, Crimea, Kabardino-Balkaria, KarachaiCherkessia, Krasnodar, Leningrad, Murmansk, Rostov, Voronezh), Serbia, Slovakia, Spain, Sweden, Switzerland, Tajikistan, Tunisia, Turkey (Adiyaman, Ankara, Antalya, Burdur, Denizli, Hakkari, Isparta, Korucuk, Muğla, Van), UK, Ukraine (Kherson, Odessa), Uzbekistan; Oriental: China; Afrotropics: Madagascar, Tanzania, Yemen, St Helena (?introduced).

Remark. Both pallipes and pseudospicatus phenotypes are present in Israel.

Syntormon pilitibia Grichanov, 2013

References: Grichanov, 2013a: 16.

Type locality: Israel: Menahamiya.

Distribution: Israel.

Syntormon subinermis (Loew, 1869)

References: Grichanov, 2007b: 150. 
Type locality: Slovakia: Losoncz [=Lučenec].

Distribution: Austria, Czech, France, Georgia, Germany, Hungary, Israel, Kyrgyzstan, Romania, Russia (Chechnya, Kabardino-Balkaria, Pskov, Vologda), Slovakia, Sweden, Tajikistan, Turkey (Adıyaman, Erzurum), Uzbekistan.

\section{Systenus Loew, 1857}

Systenus pallipes (von Roser, 1840)

References: Grichanov, 2007b: 150.

Type locality: not given (Wurttemberg) [Germany].

Distribution: Austria, Belgium, Czech, Denmark, Finland, France, Germany, Ireland, Israel, Golan Heights, Netherlands, Norway, Poland, Russia (Adygea, Krasnodar, Vladivostok, Voronezh), Slovakia, Sweden, Switzerland, Turkmenistan, UK.

Systenus vasilii Grichanov, 2002

References: Grichanov, 2002: 220.

Type locality: Mt. Hermon.

Distribution: Israel, Golan Heights.

\section{Tachytrechus Haliday, 1851}

Tachytrechus notatus (Stannius, 1831)

References: Grichanov, 2007b: 150.

Type locality: Germany: Hamburg.

Distribution: Abkhazia, Armenia, Austria, Belgium, Bosnia and Herzegovina, Bulgaria, Czech, Denmark, Finland, France, Germany, Greece incl. Crete, Hungary, Iran, Ireland, Israel, Golan Heights, Italy, Morocco, Netherlands, Norway, Poland, Romania; Russia (Adygea, Chechnya, Crimea, Kabardino-Balkaria, Krasnodar, Yakutia), Slovakia, Spain incl. Canary Is., Sweden, Switzerland, Syria; Turkey (Aydin, Korucuk, Muğla), Turkmenistan, UK.

Tachytrechus planitarsis Becker, 1907

References: Pârvu, 1997: 180; Grichanov, 2007b: 151.

Type locality: Algeria: Biskra.

Distribution: Algeria, Egypt, Iran, Israel, Morocco, Saudi Arabia, Spain (Canary Is.), Tunisia, Turkmenistan; Afrotropical: Ethiopia.

Tachytrechus tessellatus (Macquart, 1842)

References: Grichanov, 2007b: 151.

Type locality: Senegal.

Distribution: Egypt, Israel, Turkey (Antalya); Oriental: China (Taiwan), India, Indonesia, Malaysia, Philippines, Sri Lanka, Japan (Ryukyu Is.); Australasian: New Caledonia; Afrotropical: Aldabra, Angola, Botswana, Burundi, Cameroon, Cape Verde Is., DR Congo, Ethiopia, Gambia, Kenya, Madagascar, Malawi, Mauritius, Mozambique, Namibia, Nigeria, Senegal, Seychelles, Socotra, South Africa, Swaziland, Tanzania.

\section{Teuchophorus Loew, 1857}

Teuchophorus bisetus Loew, 1871

References: Grichanov, 2007b: 151.

Type locality: Uzbekistan: "Seravschan Thal, Turkestan".

Distribution: Iraq, Israel, Tajikistan, Turkey, Uzbekistan.

Teuchophorus israelensis Grichanov, Negrobov et Selivanova, 2012

References: Grichanov et al., 2012: 92.

Type locality: Israel: Park HaYarden.

Distribution: Israel.

Teuchophorus monacanthus Loew, 1859

References: Pârvu, 1996: 284; Pârvu, 1997: 180.

Type locality: not given.

Distribution: Austria, Azerbaijan, Belgium, Bulgaria, Czech, Denmark, France, Georgia, Germany, Greece incl. Crete, Hungary, Iran, Iraq, Ireland, Israel, Italy, Latvia, Luxembourg, Netherlands, Norway, Poland, Romania, Russia (Adygea, Chechnya, Kabardino-Balkaria, Krasnodar, Leningrad, Lipetsk, Murmansk, Stavropol, Voronezh), Slovenia, Spain, Sweden, Switzerland, Turkey (Antalya, Artvin, Mugla), UK, "Yugoslavia”, “Middle Asia”.

\section{Thinophilus Wahlberg, 1844}

Thinophilus flavipalpis (Zetterstedt, 1843)

References: Bodenheimer, 1937: 187 (“Palestine"); Grichanov, 2007b: 151.

Type locality: Sweden: Gottlandia, Bursviken.

Distribution: Austria, Azerbaijan, Belgium, Bulgaria, N China, Croatia, Czech, Denmark, Egypt, Estonia, Finland, France, Germany, Greece (Crete, North Aegean), Hungary, Iran, Israel, Italy, Kazakhstan, Kyrgyzstan, Moldova, 
Mongolia, Morocco, Netherlands, Poland, Portugal, Romania, S Russia (Astrakhan, Crimea, Krasnodar, Rostov), Spain, Sweden, Syria, Turkey (Ankara), UK, Ukraine (Kherson, Odessa); Oriental: China.

Thinophilus indigenus Becker, 1902

References: Grichanov, 2007b: 151.

Type locality: Egypt: Cairo, Assiur, Luxor, Assuan, Fayum, and Suez.

Distribution: Algeria, Egypt, Iran, Israel, Mongolia, Oman, Turkey; Oriental: China, India, Malaysia, Nepal, Philippines; Afrotropical: Angola, Benin, DR Congo, Cape Verde Is., Ethiopia, Gambia, Ghana, Madagascar, Namibia, Nigeria, Yemen, South Africa, Swaziland, Tanzania.

Thinophilus quadrimaculatus Becker, 1902

References: Grichanov, 2007b: 151.

Type locality: Egypt: Cairo.

Distribution: Palaearctic: Algeria, Egypt, Iran, Israel, Tajikistan, Tunisia.

Thinophilus spinitarsis Becker, 1907

References: Grichanov, 2007b: 151.

Type locality: China: "O. Zaidam, im nord-Osu; Tibet: Kurlyk am Fl. Baingol”.

Distribution: Palaearctic China, Iran, Israel, Tajikistan, Ukraine (Kherson).

\section{Thrypticus Gerstaecker, 1864}

Thrypticus bellus Loew, 1869

References: Grichanov, 2007b: 152.

Type locality: England: Kew.

Distribution: Abkhazia, Armenia, Austria, Azerbaijan, Belgium, Bosnia and Herzegovina, Bulgaria, China, Croatia, Czech, Denmark, Egypt, Finland, France, Germany, Greece, Hungary, Iran, Ireland, Israel, Golan Heights, Italy, Kazakhstan, Lithuania, Morocco, Netherlands, Poland, Romania, Russia (Krasnoyarsk, Leningrad, Novosibirsk, Rostov, Vladivostok, Voronezh), Slovakia, Spain, Sweden, Switzerland, Turkey (Erzurum, Kars, Muğla), UK, Ukraine; Afrotropical: DR Congo, Ethiopia, Kenya, Senegal, South Africa, Tanzania, St. Helena.

Thrypticus smaragdinus Gerstäcker, 1864

References: Grichanov, 2007b: 152.

Type locality: not given [Germany].

Distribution: Austria, Belgium, Croatia, Czech, Finland, France, Germany, Hungary, Israel, Netherlands, Russia (Krasnodar, Rostov, Stavropol), Sweden, Ukraine (Kherson, Odessa), UK.

Thrypticus viridis Parent, 1932

References: Grichanov, 2007b: 152.

Type locality: Germany: Berlin, Grunewald.

Distribution: France, Germany, Israel, Netherlands, Turkey (Antalya).

\section{Trigonocera Becker, 1902}

Trigonocera rivosa Becker, 1902

References: Grichanov, 2007b: 152, 2013c: 4.

Type locality: Egypt: Nile River from Luxor to Alexandria.

Distribution: Egypt, Israel; Afrotropical: Botswana, Cape Verde Is., Namibia, Senegal, Zambia; Oriental: China (Taiwan), Thailand.

\section{Conclusion}

As a result of the last two-decade investigation, a new material of Dolichopodidae was collected, identified and published in many papers; and the Israeli fauna reached to 108 species listed above. The study of the dolichopodid fauna of Israel is not finished. Several more small-sized species from unrevised genera or species groups in my hands are still awaiting description, being probably endemics to the eastern Mediterranean. There are some unverified records of Dolichopodidae from the territory (e.g., Grichanov, 2007a) and they must be confirmed. Nevertheless, an annotated checklist of Dolichopodidae (Diptera) of Israel and adjacent territories (Golan Heights and West Bank) seems worth publishing in order to facilitate future preparation of regional catalogs, keys and faunas.

At present, Sciapus with its 14 known species is the most species-rich genus in the country, followed by Medetera (12 species) and Dolichopus (10 species). Whether or not Arabshamshevia and Shamshevia Grichanov, 2012, are synonymic names (Grichanov, 2016), this is the only case of recording a non-Palaearctic dolichopodid genus in Israel. Fourteen species are now conditional endemics of this territory.

\section{References}

Becker, T. (1917). Dipterologische Studien. Dolichopodidae. Paläarktische Region. Erster Teil. Nova Acta Academiae Caesareae Leopoldinisch-Carolinae Germanicae Naturae Curiosorum, 102, 113-361. 
Grichanov, I.Ya. (2017). An annotated checklist.... Acta Biologica Sibirica, 2017, 3(4), 6-19

Bodenheimer, F.S. (1937). Prodromus Faunae Palaestine. Mémoires de I'Institut d'Égypte, 33, 1-286.

Freidberg, A. (1988). Zoogeography of the Diptera of Israel. In: Yom-Tov, Y., Tchernov, E. (Eds.). The zoogeography of Israel. Dordrecht: Dr. W. Junk Publishers, 277-308.

Gatt, P. (2012). Two new species of Microphorella Becker (Diptera: Dolichopodidae) from the Mediterranean. Revue suisse de Zoologie, 119(3), 287-302.

Grichanov, I.Ya. (2000). West-Palearctic species of the genus Ludovicius (Diptera: Dolichopodidae). Russian Entomological Journal, 9(3), 269-274.

Grichanov, I.Ya. (2001). Afrotropical Syntormon Loew and new synonyms in the genus Rhaphium Loew (Diptera: Dolichopodidae). International Journal of Dipterological Research, 12(4), 181-194.

Grichanov, I.Ya. (2002). A new species of Systenus Loew (Diptera: Dolichopodidae) from Israel. Studia Dipterologica, 9(1), 219-223.

Grichanov, I.Ya. (2004). Review of Afrotropical Dolichopodinae (Diptera: Dolichopodidae). St.Petersburg: VIZR RAAS (Plant Protection News Suppl., ISSN 1815-3682), 1-244.

Grichanov, I.Ya. (2007a). A checklist and keys to Dolichopodidae (Diptera) of the Caucasus and East Mediterranean. St.Petersburg: VIZR, 1-160 (Plant Protection News Supplements). Available from: http://www.diptera.info/downloads/Grichanov 2007b.pdf Accessed 12 November 2017.

Grichanov, I.Ya. (2007b). New records of Dolichopodidae (Diptera) from the Middle East. International Journal of Dipterological Research, 18(3), 141-153.

Grichanov, I.Ya. (2008). Systematics of the genus Epithalassius Mik, 1891 (Diptera, Dolichopodidae). Caucasian Entomological Bulletin, 4(1), 131-136.

Grichanov, I.Ya. (2010). West-Palearctic species of the genus Neurigona Rondani (Diptera: Dolichopodidae). Russian Entomological Journal, 19(3), 249-256.

Grichanov, I.Ya. (2012). New records of Dolichopodidae from the Caucasus (Diptera: Empidoidea). Cesa News, 72, 1322.

Grichanov, I.Ya. (2013a). Systematic notes on West-Palearctic species of the genus Syntormon Loew (Diptera: Dolichopodidae). In: Grichanov, I.Ya., Negrobov, O.P. (Eds.). Fauna and taxonomy of Dolichopodidae (Diptera). Collection of papers. St.Petersburg: VIZR, 3-26 (Plant Protection News Supplements). Available from: https://archive.org/details/GrichanovNegrobovDolichopodidae2013 Accessed 12 November 2017.

Grichanov, I.Ya. (2013b). Afrotropical species of the genus Asyndetus Loew (Diptera: Dolichopodidae) with notes on some Palaearctic and Oriental species. In: Grichanov, I.Ya., Negrobov, O.P. (Eds.). Fauna and taxonomy of Dolichopodidae (Diptera). Collection of papers. St.Petersburg: VIZR, 27-46 (Plant Protection News Supplements). Available from: https://archive.org/details/GrichanovNegrobovDolichopodidae2013 Accessed 12 November 2017.

Grichanov, I.Ya. (2013c). Species of the genus Trigonocera Becker (Diptera: Dolichopodidae). Journal of Insect Biodiversity, 1(6), 1-14. http://dx.doi.org/10.12976/jib/2013.1.6, http://www.insectbiodiversity.org.

Grichanov, I.Ya. (2016). Genus Arabshamshevia Naglis in the Palaearctic Region (Diptera: Dolichopodidae). Israel Journal of entomology, 46, 93-98.

Grichanov, I.Ya. (2017). Alphabetic list of generic and specific names of predatory flies of the epifamily Dolichopodoidae (Diptera). 2nd Edition. St.Petersburg: VIZR, 1-563. (Plant Protection News Supplements, N23). Available from: https://archive.org/download/Grichanov2017DolibankSec/Grichanov\%202017\%20Dolibank-sec.pdf $\quad$ Accessed 12

November 2017.

Grichanov, I.Ya., Ahmadi, A. (2017). Palaearctic species of the genus Lamprochromus Mik, 1878 (Diptera: Dolichopodidae). Far Eastern Entomologist, 336, 1-12. Available from: http://biosoil.ru/fee/Downloads/336.pdf Aaccessed 12 November 2017.

Grichanov, I.Ya., Ahmadi, A., Kosterin, O.E. (2017). New records of long-legged flies (Diptera, Dolichopodidae) from Central and North-Eastern Iran. Acta Biologica Sibirica, 3 (4), 99-112.

Grichanov, I.Ya., Kazerani, F. (2014). A new species of Sybistroma Meigen (Diptera: Dolichopodidae) from the Middle East with a key to West-Palaearctic species of the genus. Zootaxa, 3866(4), 572-582. http://dx.doi.org/10.11646/zootaxa.3866.4.7.

Grichanov, I.Ya., Negrobov, O.P. (2014). Palaearctic species of the genus Sciapus Zeller (Diptera: Dolichopodidae). St.Petersburg: VIZR, 1-84 (Plant Protection News Supplements, N13). Available from: https://archive.org/details/GrichanovNegrobov2014Sciapus Accessed 12 November 2017.

Grichanov, I.Ya., Negrobov, O.P., Selivanova, O.V. (2012). A review of Palaearctic Teuchophorus Loew (Dolichopodidae, Diptera) with an updated catalog and revised key to species. Russian Entomological Journal, 21(1), 89-96.

Kechev, M., Ivanova, M. (2015). New records of Dolichopodidae (Diptera) for Bulgaria. Comparison of dolichopodid diversity on river banks and in some forests of the Upper Thracian Plain. Silva Balcanica, 16(1), 87-104.

Negrobov, O.P. (1979). Dolichopodidae, Unterfamilie Hydrophorinae. In: Lindner, E. (Ed.). Die Fliegen der Palaearktischen Region. Stuttgart, 4 (29), 321, 419-474.

Negrobov, O.P. (1991). Dolichopodidae. In: Soos, A., Papp, L., Oosterbroeck, P. (Eds.). Catalogue of Palaearctic Diptera 7: Dolichopodidae-Platypezidae. Budapest: Akadémiai Kiadó, 1-291. DOI:10.1016/B978-0-444-98731-0.50008-9.

Negrobov, O.P., Grichanov, I.Y., Selivanova, O.V. (2013). Palearctic species of the Rhaphium albifrons group (Diptera: Dolichopodidae). Euroasian Entomological Journal, 12(6), 601-606. 
Parent, O., (1932). Sur quelques Dipteres Dolichopodidés, la plupart appartenant a la collection L. Oldenberg. Notes et description (Dipt.). Stettiner entomologische Zeitung, 93(2), 220-241.

Pârvu, C. (1996). Dolichopus siculus stat.n. (Diptera: Dolichopodidae) and some other species not mentioned in Israel [Results of the "Emil Racovita" Speleological Institution of Bucharest-Romania in Israel - 1995]. Travaux du Muséum national d'Histoire naturelle "Grigore Antipa", 36, 279-285. Available from: https://www.travaux.ro/web/pdf/36TMNHNGA-279285.pdf Accessed 12 November 2017.

Pârvu, C. (1997). New data about some species of Dolichopodidae (Diptera) from Israel (Results of "Emil Racovita" Speleological Institution of Bucharest-Romania in Israel - 1995). Travaux du Museum National d'Histoire Naturelle "Grigore Antipa", 39, 179-181.

Pârvu, C. (2002). Checklist of Dolichopodidae (Diptera) of Romania (XX). Travaux du Museum National d'Histoire Naturelle "Grigore Antipa", 44, 267-276.

Pârvu, C. (2008). Three Mediterranean Dolichopodidae (Diptera) little know [Results of some Romanian expeditions in Israel, 1995 and Morocco, 2007]. Travaux du Museum National d'Histoire Naturelle "Grigore Antipa", 51, 345-352.

Tonguç, A., Grichanov, I.Ya., Naglis, S. (2016). Checklist of the Dolichopodidae (Diptera, Brachycera) of Turkey. Turkish Journal of Zoology, 40(1), 14-26.

\section{Citation:}

Grichanov, I.Ya. (2017). An annotated checklist of Dolichopodidae (Diptera) of Israel and adjacent territories. Acta Biologica Sibirica, 3 (4), 6-19.

Submitted: 28.06.2016. Accepted: 27.08.2017

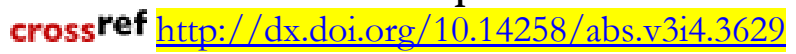

(C) 2017 by the authors. Submitted for possible open access publication under the terms and conditions of the Creative Commons Attribution (CC BY) license (http://creativecommons.org/licenses/by/4.0/). 